\title{
Clinical Translational Research
}

\section{Oral Abundance of Actinomyces spp. in Breast Cancer Patients}

\author{
Ceren Bilgilier $^{\mathrm{a}}$ Thorsten Fuereder $^{\mathrm{b}}$ Marie-Theres Kastner ${ }^{\mathrm{a}}$ \\ Zoltan Vass $^{a}$ Ingeborg Brandl ${ }^{c}$ Hanka Sahbegovic $^{c}$ Christian F. Singer $^{c}$ \\ Christoph Steininger ${ }^{a} d$
}

aDivision of Infectious Diseases and Tropical Medicine, Department of Medicine I, Medical University of Vienna, Vienna, Austria; ${ }^{b}$ Division of Oncology, Department of Medicine I, Medical University of Vienna, Vienna, Austria; 'Department of Obstetrics and Gynecology and Comprehensive Cancer Center, Medical University of Vienna, Vienna, Austria; ${ }^{\mathrm{d} K a r l-L a n d s t e i n e r ~ I n s t i t u t e ~ o f ~ M i c r o b i o m e ~ R e s e a r c h, ~ S t . ~ P o ̈ l t e n, ~ A u s t r i a ~}$

\section{Keywords}

Actinomyces spp. · Medication-related osteonecrosis of the jaw $\cdot$ Breast cancer

\begin{abstract}
Objectives: Pathophysiology of medication-related osteonecrosis of the jaw (MRONJ) is still unclear, and disease development is associated with adverse reaction of bisphosphonates and denosumab, and Actinomyces spp. as well. In this study, we evaluated the abundance of Actinomyces spp. in breast cancer patients undergoing chemotherapy compared to healthy controls. Methods: Oropharyngeal samples were collected from treatment-naive early-stage breast cancer patients, who were scheduled for standard of care therapy (eight samples throughout chemotherapy, one prior to radiotherapy and one after a year of start), as well as from healthy controls at matched timepoints. We quantified Actinomyces spp. in the samples with a highly sensitive and specific quantitative polymerase chain reaction. Results: Twenty-one patients and 16 healthy subjects were enrolled. Fortyeight percent of patients suffered from estrogen receptor-positive/progesterone receptor-positive or -negative/human epidermal growth factor receptor 2 (HER2)-neg-
\end{abstract}

Karger@karger.com www.karger.com/ocl

Karger $\stackrel{\text { ' }}{=}$
(C) 2022 The Author(s)

Published by S. Karger AG, Basel

This is an Open Access article licensed under the Creative Common Attribution-NonCommercial-4.0 International License (CC BY-NC) (http://www.karger.com/Services/OpenAccessLicense), applicable to the online version of the article only. Usage and distribution for commercial purposes requires written permission. ative disease, $38 \%$ were HER2-positive, and $14 \%$ were triplenegative. Comparison of Actinomyces spp. loads in cancer patients and healthy controls did not reveal significant difference. Fluctuations on bacterial quantity were observed in both groups over time. Tumor receptor status or different chemotherapy schemes of patients were not correlated with a particular pattern on abundance of Actinomyces spp. Conclusions: We suggest that Actinomyces spp. are not the initiative factors in MRONJ development. These bacteria are not altered in abundance during chemotherapy, but they behave opportunistic when there is a bone disruption in the orophar$y n x$ in the first place caused by antiresorptive drugs or dental trauma and proliferate in their new niche. Thus, Actinomyces spp. plays a latter role in MRONJ development, rather than a primary causative one.

(c) 2022 The Author(s)

Published by S. Karger AG, Basel

\section{Introduction}

Medication-related osteonecrosis of the jaw (MRONJ) was first described in 2003, shortly after approval of intravenous treatment with bisphosphonates, in patients with malignant diseases [1]. Several case series and observa- 
tional studies have revealed that development of MRONJ is associated with serious adverse drug reaction of bisphosphonates and the receptor activator of nuclear factor kappa B ligand inhibitor, denosumab [2]. Incidence of this severe debilitating condition ranges between 0.7 and $18 \%$ in cancer patients according to recent studies [2-5].

Bisphosphonates and denosumab are two different classes of antiresorptives that are prescribed as the most effective antiresorptive drugs to treat (malignant) hypercalcemia and to reduce the incidence of skeletal-related events in patients with bone metastases [6-9]. Despite the benefits of these drugs in oncology, they were identified as significant risk factors for MRONJ when given long term and in high dosage $[10,11]$. Antiangiogenic drugs were associated with MRONJ development as well [12].

The pathophysiology of MRONJ cannot be fully explained by a single model. Main characteristics of MRONJ lesions are scattered areas of exposed or nonhealing necrotic bone in the maxillofacial region persisting for more than 8 weeks and is often accompanied by local infection of the necrotic bone $[13,14]$. The unique localization of the jaw in MRONJ is explained by the higher susceptibility of jawbone to infections compared to other bones in the body, considering exposure to millions of bacteria in the oral cavity [15]. In case the mucosal barrier is breached due to trauma or surgical procedures during or after bisphosphonates or denosumab treatment, emerging infection causes lower $\mathrm{pH}$ in that area, which ultimately leads to suppression of the healing process and thereof bone necrosis $[9,16]$. The most common precipitating factor for osteonecrosis is tooth extraction, suggested by $52-61 \%$ of MRONJ patients $[15,17]$.

In recent years, small case series and reviews suggested a link between evolution of MRONJ and Actinomyces species [18-22]. Actinomyces spp. were found in $89 \%$ and $82 \%$ of bone samples from MRONJ patients in two recent retrospective studies by Russmüller et al. [16] and Cerrato et al. [23], respectively. Clinical relevance of the family of Actinomycetaceae is progressively recognized, and more than 40 species identified [24]. Actinomyces spp. are ubiquitous Gram-positive, facultative anaerobic (except A. meyeri and $A$. israelii that are obligate anaerobes), nonspore-forming bacteria, and they are commensals in the skin, oral, gut, and vaginal flora [16].

Oral microbiota is altered during chemotherapy in patients with cancer, and the alterations may lead to severe local or systemic pathologies [25]. Mucositis and oral candidiasis are common nonhematological complications of cancer chemotherapy, range from $40-80 \%$ to $7-52 \%$ in cancer patients, respectively $[26,27]$. In a recent study by Hong et al. [28], oral bacteriome disruption associated with mucositis during chemotherapy was investigated, and they represented a negative correlation between severity of mucositis and salivary bacterial abundance of Streptococcus, Veillonella, and Actinomyces species.

We revealed that the largest group within MRONJ patients consists of breast and prostate cancer patients, in a previous retrospective study [16]. Thereof we chose earlystage breast cancer patients, who were treatment-naive and did not receive bisphosphonates or denosumab, and collected oropharyngeal samples from them for a year throughout their therapies. It was the aim of this study to determine the fluctuations of Actinomyces spp. quantity during chemotherapy and compare the abundance of $A c$ tinomyces spp. in early-stage breast cancer patients to healthy individuals at matched timepoints.

\section{Materials and Methods}

\section{Patients and Samples}

This study was conducted with a total of 37 female patients who were older than 18 years. Of the 37 patients, two groups were formed with 21 early-stage treatment-naive breast cancer patients (mean age $51.7 \pm 11$ years) scheduled for neoadjuvant chemotherapy and 16 healthy controls (mean age $50.2 \pm 12.5$ years). The patients were excluded from the study if they (a) had a metastatic disease and/or received chemotherapy previously; (b) received antimicrobial drugs within 1 month prior to enrollment; (c) received bisphosphonates or denosumab, oral hormone contraceptives, and immunosuppressive treatments within 3 months prior to enrollment; (d) had invasive dental procedures within 3 months prior to enrollment; and (e) had known endocrine disorder requiring treatment.

Clinical data collection included patients' age, ECOG performance status, medical history, body mass index, clinical stage, histological subtype of carcinoma, estrogen receptor (ER), progesterone receptor (PR) and human epidermal growth factor receptor 2 (HER2) status of tumor, chemotherapy cycles administered, and adverse events during chemotherapy such as mucositis or oral candidiasis. Oropharyngeal lavage samples were collected at 10 timepoints from each patient. These were the days of each chemotherapy cycle with approximately 3 weeks of intervals (starting from day 1 and continues with cycles 2-8), prior to radiotherapy (preRadio; or on a matching day if the patient did not need radiotherapy), and at the end of a year (week 52) for early-stage breast cancer patients. Samples were collected from healthy individuals as well at matched timepoints. The samples were stored at $-80^{\circ} \mathrm{C}$ until the microbial evaluation, and all samples from the same patient were thawed and processed further in the same batch.

\section{Microbial Evaluation}

An Actinomyces spp.-specific quantitative polymerase chain reaction (qPCR) was developed by using the DNA isolated from pure bacterial cultures of the six most abundant species of Actino- 
Table 1. Clinical data of breast cancer patients enrolled in the study

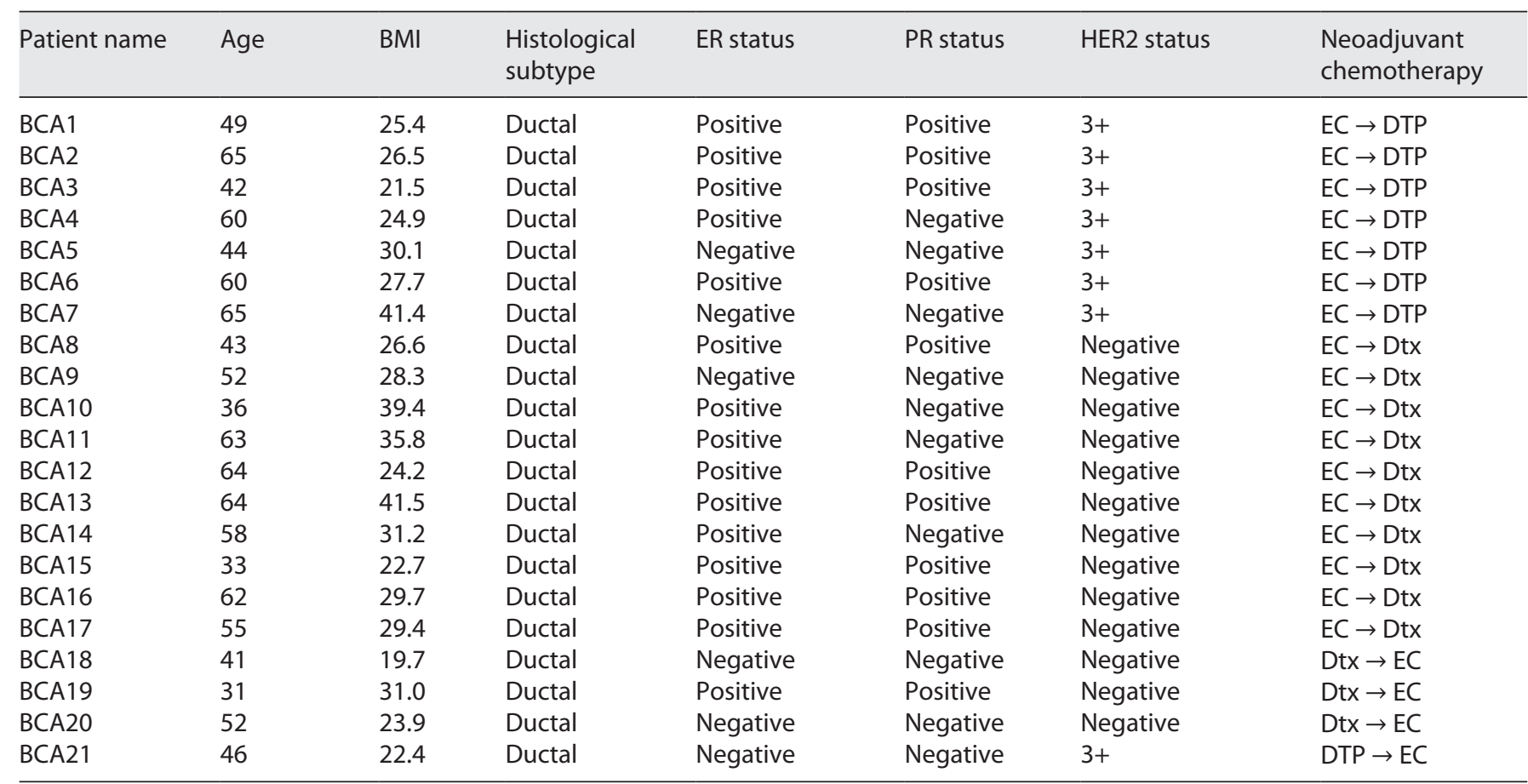

Neoadjuvant chemotherapy was scheduled for 8 cycles (4 + 4). BMI, body mass index; ER, estrogen receptor; PR, progesterone receptor; EC, epirubicin + cyclophosphamide; DTP, docetaxel + trastuzumab + pertuzumab; Dtx, docetaxel.

myces in the oral cavity; A. turicensis, A. meyeri, A. neuii, A. odontolyticus, A. europaeus, and $A$. naeslundii. Sensitivity of the assay was assessed with spiking experiments, in which different predefined concentrations of bacteria were introduced to naive samples. The primers (adapted from Bizhang et al. [29]) and the probe used 16S rRNA gene amplification and was designed as following: Act-fw: 5' - GGTCTCTGGGCCGTTACTGA -3', Act-rv: 5'CCCCCACACCTAGTGCCC -3', and Act-probe: 5'- FamCGTGGGGAGCGAACAGGATTAGATACC-Tamra - $3^{\prime}$.

Genomic DNA (gDNA) was isolated from the oropharyngeal lavage samples of 21 breast cancer patients and 16 healthy individuals with the use of QIAsymphony DSP Virus/Pathogen Kit (QIAGEN, Hilden, Germany), following the manufacturer's recommendations. The quality and concentration of each gDNA sample were measured by the NanoDrop spectrophotometer (Thermo Fisher Scientific, Waltham, MA, USA), and to normalize the amount of gDNA used in qPCR, the samples were diluted to an end concentration of $10 \mathrm{ng} / \mu \mathrm{L}$ gDNA. The reaction mixture consisted of $10 \mu \mathrm{L}$ iTaq Universal Probes Supermix (Bio-Rad, Hercules, CA, USA), $1.1 \mu \mathrm{M}$ of both forward and reverse primers, 0.25 $\mu \mathrm{M}$ of the probe, and $1 \mu \mathrm{L}$ of gDNA extract made up to $20 \mu \mathrm{L}$ with water. The cycling protocol was designed according to manufacturer's recommendations that follows as polymerase activation and initial DNA denaturation at $95^{\circ} \mathrm{C}$ for $2 \mathrm{~min}$, and 40 cycles of denaturation at $95^{\circ} \mathrm{C}$ for $15 \mathrm{~s}$ and primer annealing and extension at $65^{\circ} \mathrm{C}$ for $1 \mathrm{~min}$. As positive control and negative controls in qPCR, A. odontolyticus DNA that was previously isolated from pure bacterial culture and distilled water was added to the master mix, respectively. All samples were run in duplicates.

Actinomyces spp. Load in Breast Cancer

Patients
All samples from the same patient were run in the same Actinomyces spp.-specific qPCR, and the Ct value of the positive control was noted. Considering that $\mathrm{Ct}$ value of positive control remained equal in each qPCR run, we calculated the $\Delta \mathrm{Ct}$ values and fold change by $2^{-\Delta \Delta C t}$ method as described before [30]. Abundance of bacteria is inversely correlated with $\Delta \mathrm{Ct}$ and positively correlated with fold change.

\section{Statistical Analysis}

We assessed statistical analysis of our Actinomyces spp.-specific qPCR results with the use of GraphPad Prism 9 (GraphPad Software, Inc., San Diego, CA, USA) and ran 2-tailed tests or 2-way ANOVA multiple comparison tests, as appropriate. Significance was determined by $p$ values $<0.05$.

\section{Results}

Clinical data of the 21 breast cancer patients were included in this study, and immunohistochemistry results of their biopsy samples are described in Table 1. Patients were divided into three groups based on tumor receptor status; (i) $\mathrm{ER}+/ \mathrm{PR}+/ \mathrm{HER} 2-$ or ER+/PR-/HER2- (10/21 patients), (ii) $\mathrm{ER}+/ \mathrm{PR}+/ \mathrm{HER} 2+, \mathrm{ER}-/ \mathrm{PR}-/ \mathrm{HER} 2+$ or $\mathrm{ER}+/ \mathrm{PR}-/$ HER2 + (8/21 patients), and (iii) triple-negative: ER-/PR-/ HER2- (3/21 patients). Different chemotherapy regimens 


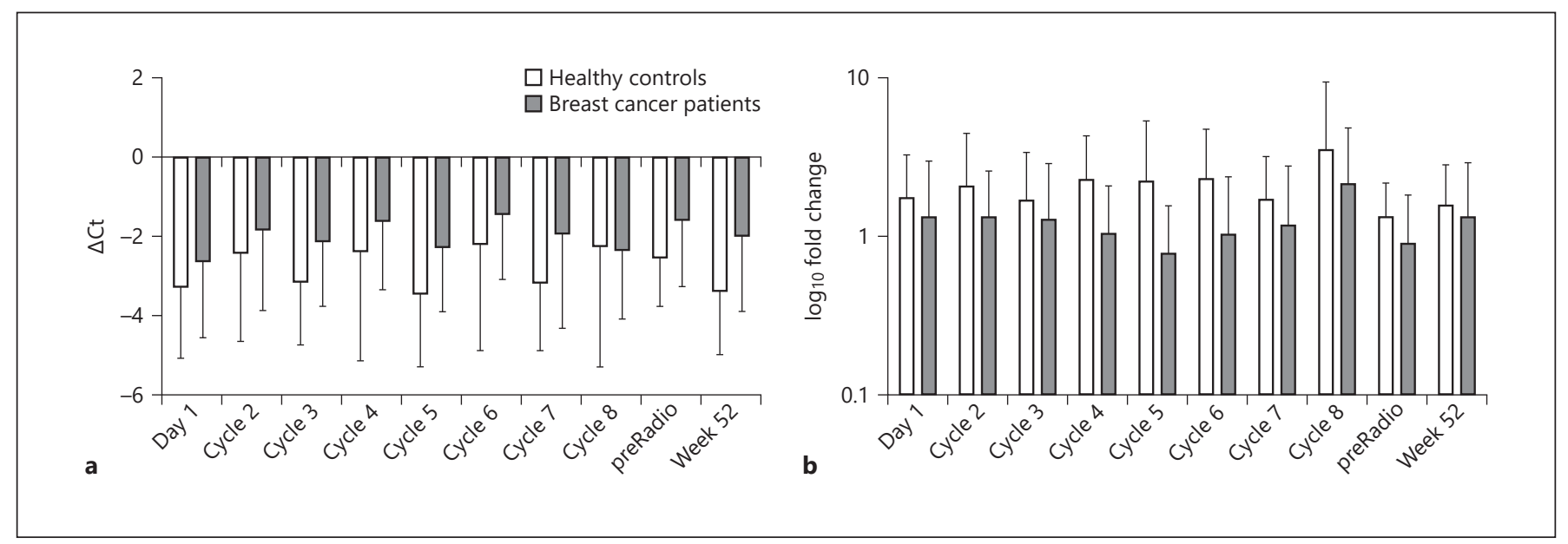

Fig. 1. Quantification of Actinomyces spp. in oropharyngeal samples of breast cancer patients during chemotherapy and healthy individuals at matched timepoints. Columns represent the mean of the group at a given timepoint, and standard deviations are in- dicated by error bars. Abundance of bacteria is inversely correlated with $\Delta \mathrm{Ct}$ (a) and positively correlated with fold change (b). No significant difference was observed between two groups at any time.
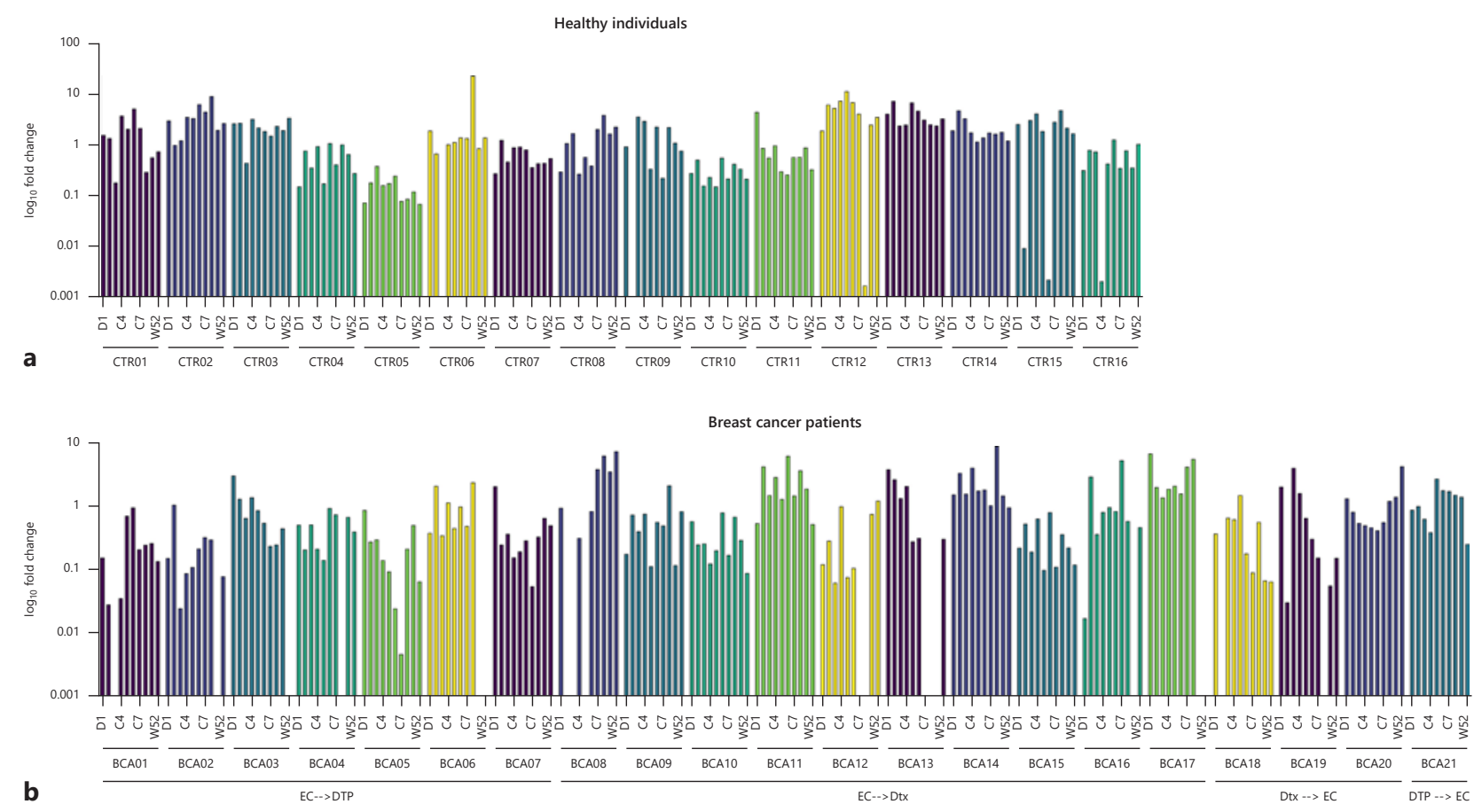

Fig. 2. Bacterial loads of Actinomyces spp. in oropharyngeal samples of healthy individuals (a) and breast cancer patients (b) on a single patient level. Each column represents a timepoint of collection, respectively. In the chart of breast cancer patients, the chemotherapy schemes of the patients are indicated below. Chemo- therapeutical drugs were prescribed in different schemes for patients for their first four and last four cycles in therapy. EC, epirubicin + cyclophosphamide; DTP, docetaxel + trastuzumab + pertuzumab; Dtx, docetaxel; D1, day 1; C2-8, cycles $2-8$, prior to radiotherapy; W52, week 52; preRadio, prior to radiotherapy. 
were prescribed to breast cancer patients according to their tumor biology for routine purposes; $17 / 21$ patients received epirubicin + cyclophosphamide in the first four cycles and continued with either only docetaxel (Dtx) or Dtx + trastuzumab + pertuzumab ( $n=10$ and $n=7$, respectively) in the next four cycles, while $4 / 21$ patients received these drugs the other way round ( $n=3$ and $n=1$, respectively). Surprisingly, none of the cancer patients develop mucositis or oral candidiasis during chemotherapy. Neither breast cancer patients nor healthy controls used antibiotics or visited a dentist during the time period of oropharyngeal sample collection for a year.

Amplification of Actinomyces spp.-specific genes from gDNA extracted from oropharyngeal samples of breast cancer patients and healthy controls at different timepoints is shown in Figure 1. Actinomyces spp. were more abundant in the samples of healthy individuals at all timepoints, with an insignificant difference to breast cancer patients $(p>0.05)$. We observed variations of the bacterial loads of Actinomyces spp. during chemotherapy cycles in breast cancer patients; however, these differences were also not significant. A similar fluctuation of bacterial loads was monitored in healthy controls as well.

The levels of Actinomyces spp. in samples collected from all patients enrolled in this study are represented in Figure 2, on a timeline. Bacterial loads were not correlated with age ( $p=0.852)$, body mass index $(p=0.242)$, or tumor receptor status $(p=0.093)$ at day 1 . Over the period of a year, changes on bacterial loads of Actinomyces spp. were observed in both groups. Since only one sample per timepoint was collected from the patients and gDNA was extracted once, it is not reliable to make statistical analysis on abundance of Actinomyces spp. compared between timepoints on a patient level. However, when all patients in the same group observed together, a trend could be seen. In our study, we did not observe a specific trend on abundance of Actinomyces spp. in earlystage breast cancer patients over their chemotherapies. Considering the different chemotherapy schemes prescribed to breast cancer patients, we also did not record a specific pattern with any treatment option (Fig. 2b).

\section{Discussion}

Increasing cases of MRONJ have been reported in the last years; nevertheless, we still could not decipher the underlying mechanism clearly. It is urgently necessary to develop competent prophylactic and therapeutic strategies since only $30 \%$ of MRONJ patients may be treated by cur-

Actinomyces spp. Load in Breast Cancer Patients rently applications [31]. Data on MRONJ published so far suggest three different, successive factors for the development of the disease: (a) changes in bone metabolism leading to a reduced tendency of spontaneous healing of bone and mucosa; these changes may be associated with antiresorptive drugs but also underlying malignant disease, hormone disorders or treatments, genetic polymorphisms, etc.; (b) exposure of the bone; for instance, by mucositis induced by chemotherapy, poor oral hygiene, or tooth extraction; and (c) the presence of Actinomyces spp. in high quantities in the oropharynx that allows the bacteria to enter the exposed bone in significant numbers to overwhelm host immunity and cause local infection [13-15, 17]. In this study, our focus was on the latter factor so that we investigated the abundance of Actinomyces spp. in early-stage breast cancer patients as a leading patient group in MRONJ development.

We monitored the bacterial loads of Actinomyces spp. throughout the chemotherapy of breast cancer patients, compared to healthy controls, in order to investigate if these bacteria have an altered abundance in cancer patients in the first place, or if the chemotherapeutical drugs have an effect on Actinomyces spp. in quantity. In order to eliminate biases of that, use of bisphosphonates and denosumab has been associated with MRONJ development, together with the presence of Actinomyces spp. in the oral cavity, and we only chose the breast cancer patients who did not receive any of these drugs in their treatment. Patients enrolled in this study, either to the breast cancer or control group, did not use antimicrobial drugs during the time of sample collection for a year. Since early-stage breast cancer patients received 3-weekly chemotherapy and an oropharyngeal sample was collected before each cycle, the healthy individuals were invited to donate samples every 3 weeks, as well. A final sample was collected from both groups at the end of the year to indicate if there is a consistent effect of chemotherapeutical drugs on Actinomyces spp. levels.

Abundance of Actinomyces spp. in the oral cavity fluctuated over time both in breast cancer patients and healthy controls, without any specific pattern. We did not observe an influence of chemotherapeutical drugs (epirubicin, cyclophosphamide, docetaxel, trastuzumab, and pertuzumab) on bacterial loads as well. Yet, these findings should be interpreted with caution since none of the cancer patients included in this study developed mucositis during chemotherapy. Oral mucositis has been associated with alterations in oral microbiome so that due to lack of mucositis and small cohort size in this study, there might be biases.

When bacterial loads of Actinomyces spp. in oropharyngeal samples of breast cancer patients and healthy controls were compared, there was no significant differ- 
ence. Despite not being significant, Actinomyces spp. were more abundantly found in healthy donors' samples at all timepoints. Thus, it is tempting to speculate that early-stage breast cancer patients might have a dysbiotic gut microbiota, and some other types of commensal bacteria could have surpassed Actinomyces spp.. Multiple studies published in recent years showed an association between dysbiosis of gut microbiota leading to changes in estrogen mechanism and hormone-related diseases such as breast cancer $[32,33]$. The phyla Proteobacteria, Firmicutes, and Bacteroidetes are the most abundant bacteria in samples of breast cancer patients [34-36].

We suggest that Actinomyces spp. are not the initiative factors in MRONJ development, and they are rather the opportunistic bacteria that proliferate when they find a new niche. Only after a complication such as disruption of the bone, Actinomyces spp. may invade the maxilla or mandible and facilitate an infection by overwhelming host immunity with increased quantity in the oropharynx, as also suggested by Naik and Russo [37]. As a future aspect, abundance of Actinomyces spp. may be investigated in cancer patients who received antiresorptive drugs throughout the chemotherapy. Thereof, the phases where bacterial loads of Actinomyces spp. are increased may be determined, and with this knowledge, we may suggest antimicrobial treatment at particular stages in chemotherapy to prevent development of MRONJ.

\section{Acknowledgments}

We thank to Prof. Athanasios Makristathis and Brigitte Selitsch (Medical University of Vienna, Vienna, Austria) for providing bacterial cultures of A. turicensis, A. meyeri, A. neuii, A. odontolyticus, A. europaeus, and A. naeslundii for spiking experiments in qPCR optimization and sharing their expertise.

\section{Statement of Ethics}

This study protocol was reviewed and approved by the local Ethics Committee at Medical University of Vienna, approval number EK1539/2016. The study was performed in accordance with the Declaration of Helsinki and good practical guidelines. A written informed consent was obtained from all patients enrolled in the study.

\section{Conflict of Interest Statement}

The authors have no conflicts of interest to declare.

\section{Funding Sources}

This study was supported by Karl-Landsteiner Institute of Microbiome Research and an unconditional research grant by AMGEN.

\section{Author Contributions}

C.B. performed experiments and data analysis and wrote the manuscript. T.F. contributed to guidance and critically revised the manuscript. M.-T.K., Z.V., I.B., and H.S. provided technical assistance and collected clinical samples. C.F.S. contributed to guidance. C.S. designed and supervised the study.

\section{Data Availability Statement}

All data generated or analyzed during this study are included in this article. Further inquiries can be directed to the corresponding author.

\section{References}

1 Marx RE. Pamidronate (aredia) and zoledronate (zometa) induced avascular necrosis of the jaws: a growing epidemic. J Oral Maxillofac Surg. 2003;61:1115-7.

2 Edwards BJ, Gounder M, McKoy JM, Boyd I, Farrugia M, Migliorati C, et al. Pharmacovigilance and reporting oversight in US FDA fasttrack process: bisphosphonates and osteonecrosis of the jaw. Lancet Oncol. 2008;9:1166-72.

3 Limones A, Sáez-Alcaide LM, Díaz-Parreño SA, Helm A, Bornstein MM, MolineroMourelle P. Medication-related osteonecrosis of the jaws (MRONJ) in cancer patients treated with denosumab vs. zoledronic acid: a systematic review and meta-analysis. Med Oral Patol Oral Cir Bucal. 2020;25:e326-36.
4 Galis B, Zajko J, Hirjak D, Vanko L, Kupcova I, Jurkemik J, et al. Is the prevalence of the medication-related osteonecrosis of the jaws underestimated, evaluation in oncological and non-oncological disease. Bratisl Lek Listy. 2017;118:724-31.

5 Peer A, Khamaisi M. Diabetes as a risk factor for medication-related osteonecrosis of the jaw. J Dent Res. 2015;94:252-60.

6 Saad F, Chi K, Fleshner N. The role of bisphosphonates in the management of bone metastases in prostate cancer. Can J Urol. 2004;11: 2376-82.

7 Lerman MA, Xie W, Treister NS, Richardson PG, Weller EA, Woo SB. Conservative management of bisphosphonate-related os- teonecrosis of the jaws: staging and treatment outcomes. Oral Oncol. 2013;49:97783.

8 Voss PJ, Poxleitner P, Schmelzeisen R, Stricker A, Semper-Hogg W. Update MRONJ and perspectives of its treatment. J Stomatol Oral Maxillofac Surg. 2017;118:232-5.

9 Shibahara T. Antiresorptive agent-related osteonecrosis of the jaw (ARONJ): a twist of fate in the bone. Tohoku J Exp Med. 2019;247: 75-86.

10 Bamias A, Kastritis E, Bamia C, Moulopoulos LA, Melakopoulos I, Bozas G, et al. Osteonecrosis of the jaw in cancer after treatment with bisphosphonates: incidence and risk factors. J Clin Oncol. 2005;23:8580-7. 
11 Aragon-Ching JB, Ning YM, Chen CC, Latham L, Guadagnini JP, Gulley JL, et al. Higher incidence of osteonecrosis of the jaw $(\mathrm{ONJ})$ in patients with metastatic castration resistant prostate cancer treated with anti-angiogenic agents. Cancer Invest. 2009;27:2216.

12 Kuroshima S, Sasaki M, Sawase T. Medication-related osteonecrosis of the jaw: a literature review. J Oral Biosci. 2019;61:99-104.

13 Khan AA, Morrison A, Hanley DA, Felsenberg D, McCauley LK, O’Ryan F, et al. Diagnosis and management of osteonecrosis of the jaw: a systematic review and international consensus. J Bone Miner Res. 2015;30:3-23.

14 AlDhalaan NA, BaQais A, Al-Omar A. Medication-related osteonecrosis of the jaw: a review. Cureus. 2020;12:e6944.

15 Ruggiero SL, Dodson TB, Fantasia J, Goodday R, Aghaloo T, Mehrotra B, et al. American Association of Oral and Maxillofacial Surgeons position paper on medication-related osteonecrosis of the jaw: 2014 update. J Oral Maxillofac Surg. 2014;72:1938-56.

16 Russmueller G, Seemann R, Weiss K, Stadler V, Speiss M, Perisanidis C, et al. The association of medication-related osteonecrosis of the jaw with Actinomyces spp. infection. Sci Rep. 2016;6:31604.

17 Kuroshima S, Sasaki M, Murata H, Sawase T. Medication-related osteonecrosis of the jawlike lesions in rodents: a comprehensive systematic review and meta-analysis. Gerodontology. 2019;36:313-24.

18 De Ceulaer J, Tacconelli E, Vandecasteele SJ. Actinomyces osteomyelitis in bisphosphonate-related osteonecrosis of the jaw (BRONJ): the missing link? Eur J Clin Microbiol Infect Dis. 2014;33:1873-80.

19 Lee CY, Pien FD, Suzuki JB. Identification and treatment of bisphosphonate-associated actinomycotic osteonecrosis of the jaws. Implant Dent. 2011;20:331-6.
20 Schipmann S, Metzler P, Rössle M, Zemann $\mathrm{W}$, von Jackowski J, Obwegeser JA, et al. Osteopathology associated with bone resorption inhibitors: which role does Actinomyces play? A presentation of 51 cases with systematic review of the literature. J Oral Pathol Med. 2013;42:587-93.

21 Badros A, Weikel D, Salama A, Goloubeva O, Schneider A, Rapoport A, et al. Osteonecrosis of the jaw in multiple myeloma patients: clinical features and risk factors. J Clin Oncol. 2006;24:945-52.

22 Yahara H, Hiraki A, Maruoka Y, Hirabayashi A, Suzuki M, Yahara K. Shotgun metagenome sequencing identification of a set of genes encoded by Actinomyces associated with medication-related osteonecrosis of the jaw. PLoS One. 2020;15:e241676.

23 Cerrato A, Zanette G, Boccuto M, Angelini A, Valente M, Bacci C. Actinomyces and MRONJ: a retrospective study and a literature review. J Stomatol Oral Maxillofac Surg. 2021; 122(5):499-504.

24 Könönen E, Wade WG. Actinomyces and related organisms in human infections. Clin Microbiol Rev. 2015;28:419-42.

25 Villafuerte KRV, Martinez CJH, Dantas FT, Carrara HHA, Dos Reis FJC, Palioto DB. The impact of chemotherapeutic treatment on the oral microbiota of patients with cancer: a systematic review. Oral Surg Oral Med Oral Pathol Oral Radiol. 2018:125:552-66.

26 Chaveli-López B, Bagán-Sebastián JV. Treatment of oral mucositis due to chemotherapy. J Clin Exp Dent. 2016;8:e201-9.

27 Jayachandran AL, Katragadda R, Thyagarajan R, Vajravelu L, Manikesi S, Kaliappan S, et al. Oral candidiasis among cancer patients attending a tertiary care hospital in Chennai, South India: an evaluation of clinicomycological association and antifungal susceptibility pattern. Can J Infect Dis Med Microbiol. 2016;2016:8758461.

28 Hong BY, Sobue T, Choquette L, Dupuy AK, Thompson A, Burleson JA, et al. Chemotherapy-induced oral mucositis is associated with detrimental bacterial dysbiosis. Microbiome. 2019;7:66.
29 Bizhang M, Ellerbrock B, Preza D, Raab W, Singh P, Beikler T, et al. Detection of nine microorganisms from the initial carious root lesions using a TaqMan-based real-time PCR. Oral Dis. 2011;17:642-52.

30 Livak KJ, Schmittgen TD. Analysis of relative gene expression data using real-time quantitative PCR and the 2(-delta delta $\mathrm{C}(\mathrm{T})$ ) method. Methods. 2001;25:402-8.

31 Saad F, Brown JE, Van Poznak C, Ibrahim T, Stemmer SM, Stopeck AT, et al. Incidence, risk factors, and outcomes of osteonecrosis of the jaw: integrated analysis from three blinded active-controlled phase III trials in cancer patients with bone metastases. Ann Oncol. 2012;23:1341-7.

32 Fernández MF, Reina-Pérez I, Astorga JM, Rodríguez-Carrillo A, Plaza-Díaz J, Fontana L. Breast cancer and its relationship with the microbiota. Int J Environ Res Public Health. 2018;15:1747.

33 Parida S, Sharma D. The power of small changes: comprehensive analyses of microbial dysbiosis in breast cancer. Biochim Biophys Acta Rev Cancer. 2019;1871:392-405.

34 Urbaniak C, Cummins J, Brackstone $M$, Macklaim JM, Gloor GB, Baban CK, et al. Microbiota of human breast tissue. Appl Environ Microbiol. 2014;80:3007-14.

35 Goedert JJ, Hua X, Bielecka A, Okayasu I, Milne GL, Jones GS, et al. Postmenopausal breast cancer and oestrogen associations with the IgA-coated and IgA-noncoated faecal microbiota. Br J Cancer. 2018;118:471-9.

36 Chan AA, Bashir M, Rivas MN, Duvall K, Sieling PA, Pieber TR, et al. Characterization of the microbiome of nipple aspirate fluid of breast cancer survivors. Sci Rep. 2016;6: 28061.

37 Naik NH, Russo TA. Bisphosphonate-related osteonecrosis of the jaw: the role of actinomyces. Clin Infect Dis. 2009;49:1729-32. 